\title{
ON PANOV'S THEOREM
}

\author{
PETER S. LANDWEBER ${ }^{1}$
}

Abstract. We give a simple proof of Panov's theorem, which determines the elements of $H_{*}(M U)$ mapped into $\pi_{*}(M U)$ by all operations $s_{\omega}$ for $\omega>0$.

The purpose of this note is to present a simple proof of the main theorem of N. V. Panov's paper Characteristic numbers in $U$-theory [6]. In addition, Panov goes on to obtain complete results concerning the Chern numbers of $(U, f r)$-manifolds; see $\$ 4$ of [6].

Let $M U_{*}(X)$ denote the complex bordism of a space or spectrum $X$. Then there are stable operations

$$
s_{\omega}: M U_{*}(X) \rightarrow M U_{*}(X)
$$

for each partition $\omega$; if $\omega$ is a partition of $n(|\omega|=n)$ then $s_{\omega}$ lowers degrees by $2 n([4],[5])$. We shall always assume that $|\omega|>0$ when dealing with the operations $s_{\omega}$ (for $\omega=0, s_{\omega}$ is the identity, which is of no interest here). An element $a \in M U_{*}(X)$ is called primitive if $s_{\omega}(a)=0$ for all $\omega$.

We may regard $\pi_{*}(M U)=M U_{*}$ as a submodule of $H_{*}(M U)$ by means of the Hurewicz homomorphism. If $H$ denotes the integral EilenbergMac Lane spectrum and $S$ the sphere spectrum, then

$$
H_{*}(M U) / M U_{*} \approx M U_{*}(H / S) \text {. }
$$

Let $N_{1}=\left\{a \in H_{*}(M U) ; s_{\omega} a \in M U_{*}\right.$ for all $\left.\omega\right\}$; then $N_{1} / M U_{*}$ can be identified with the primitive elements in $M U_{*}(H / S)$. Panov gives a determination of $N_{1}$.

Theorem (Panov). Let $n>0$. Then $\left(N_{1}\right)_{2 n} / M U_{2 n}$ is a cyclic group. Moreover,

(a) for $n$ odd, it has order 2 with generator $C P(1)^{n} / 2$;

(b) for $n=2$, it has order 12 with generators $C P(1)^{2} / 4$ and $C P(2) / 3$;

(c) for $n$ even and $n>2$, it has $p$-torsion iff $n \equiv 0 \bmod (p-1)$. For such $p$ write $k=n /(p-1)=p^{m} l,(p, l)=1$. If $p$ is odd, the $p$-component has order $p^{m+1}$ and generator $C P(p-1)^{k} / p^{m+1}$. If $p=2$, the 2-primary part has order

Received by the editors May 30, 1973.

AMS (MOS) subject classifications (1970). Primary 55B20.

Key words and phrases. Complex bordism theory, $M U$-characteristic numbers.

${ }^{1}$ Research supported in part by NSF Grant GP-21064. 
$2^{m+2}$ and generator

$$
C P(1) / 2^{m+2}+H_{3} C P(1)^{n-3} / 2
$$

where $H_{3}$ is a suitable generator of $M U_{6}\left(e . g ., H_{3}=2 C P(3)+H_{2,2}\right)$.

REMARKS 1. In fact Buhštaber ([1], [2]) introduces a filtration

$$
M U_{*}=N_{0} \subset N_{1} \subset N_{2} \subset \cdots \subset H_{*}(M U)
$$

where $N_{i+1} / N_{i}$ is the module of primitive elements in $H_{*}(M U) / N_{i}$. This explains the notation.

2. Let $s_{\omega}$ denote characteristic numbers in $K$-theory, in particular when $\omega$ is empty $s_{\omega}=T d$, the Todd genus. The following is Panov's Proposition 6.

Proposition. $N_{1}$ consists of those elements in $H_{*}(M U)$ for which $\boldsymbol{s}_{\omega}$ is integral for every $\omega>0$.

Panov overlooked the following simple proof. First, if $a \in N_{1}$ and $\omega>0$, then $s_{\omega}(a)=T d s_{\omega}(a) \in Z$ as desired. Conversely, let $a \in H_{*}(M U)$ have all $s_{\omega}(a)$ integral for $\omega>0$. To show $a \in N_{1}$, fix $\omega_{0}>0$; we will show $s_{\omega_{0}}(a) \in M U_{*}$. In view of the Hattori-Stong theorem, it suffices to show $s_{\omega_{1}} \circ s_{\omega_{0}}(a) \in Z$ for all $\omega_{1} \geqq 0$. This is the same as $\operatorname{Td}\left(s_{\omega_{1}} \circ s_{\omega_{0}}(a)\right)$. Since $s_{\omega_{1}} \circ s_{\omega_{0}}$ is a linear combination of $s_{\omega}$ 's with $\omega>0$ ([4], [5]), this is clear.

3 . One concludes that Panov could just as well use $K$-theory characteristic numbers. For example, at no point are the operations $s_{\omega}$ composed.

4. Panov does not pick his generator $H_{3}$ properly, since $s_{3}\left(H_{1,3}\right)$ is 0 and not -4 . A suitable choice is $H_{3}=2 C P(3)+H_{2,2}$, since $s_{3}\left(H_{3}\right)=2$ and $H_{3}$ agrees with $H_{2,2}$ mod 2 (see Proposition 3 and the preceding paragraph in [6]).

5. The evident $B P$ analogue of Panov's theorem is valid. One simply replaces $C P(p-1)$ with $v_{1}$, and $H_{3}$ with $v_{2}$ in the interesting case $p=2$ and $n$ even, $n>2$.

We now outline our proof of Panov's theorem.

(a) Show $\left(N_{1}\right)_{2 n} / M U_{2 n}$ is cyclic and has $p$-torsion iff $n \equiv 0 \bmod (p-1)$.

(b) For such $p$, write $n=(p-1) k$ and $k=p^{m} l$ with $(p, l)=1$. Show that $C P(p-1)^{k} / p^{m+1}$ lies in $\left(N_{1}\right)_{2 n}$, and unless $p=2$ and $n$ is even and $n>2$ it generates the p-component.

(c) If $n$ is even and $n>2$, show that $P=C P(1)^{n} / 2^{m+2}+H_{3} C P(1)^{n-3} / 2$ lies in $\left(N_{1}\right)_{2 n}$ and generates the 2-component of $\left(N_{1}\right)_{2 n} / M U_{2 n}$.

By proving (a) first, one avoids the need for Panov's splittings. (b) is included in Proposition 4 of [6]. Our main contribution is in the proof of (c).

At this point it is convenient to follow [6] and choose generators $\left\{H_{n}\right\}$ for $M U_{*}, H_{n} \in M U_{2 n}$, such that $H_{p-1}=C P(p-1)$ and, if $k>0$, 
$H_{p^{k+1}-1}$ is a nonzero multiple mod $p$ of Stong's hypersurface ([3], [7])

$$
H_{p^{k}}, \cdots, p^{k} \subset C P\left(p^{k}\right) \times \cdots \times C P\left(p^{k}\right)
$$

(where $p^{k}$ occurs $p$ times). In particular, $H_{3} \equiv H_{2,2} \bmod 2$.

For a fixed prime $p$ and $a \in N_{1}$, we say that $a$ has type $\omega_{0}$ if $s_{\omega_{0}}(a) \not \equiv$ $0 \bmod p$ and $s_{\omega}(a) \equiv 0 \bmod p$ if $\omega>\omega_{0}$. Recall that one puts $\omega>\omega_{0}$ if $|\omega|>\left|\omega_{0}\right|$, or if $|\omega|=\left|\omega_{0}\right|$ and $\omega$ involves fewer terms than $\omega_{0}$; see [3], [7]. For example, $H_{p-1}$ has type $0 ; H_{p^{k+1}-1}$ has type $\left(p^{k}-1, \cdots, p^{k}-1\right)$ if $k>0$ (where $p^{k}-1$ occurs $p$ times); and $H_{n}$ has type $(n)$ if $n+1$ is not a power of $p$. For the $H_{p^{k+1}-1}$ this is due to Stong ([3], [7]) if one computes with $K$-theory characteristic numbers. The Chern-Dold character makes it possible to carry over the computation to $M U$-characteristic numbers; see Proposition 2 of [6].

Notice that one has $s_{\omega} C P(1)^{n}=0$ if $\omega \neq(1, \cdots, 1)$. As is customary, write $s_{\omega}=s^{k \Delta_{1}}$ if $\omega=(1, \cdots, 1)$ with $k$ l's. Then we have

$$
s^{k \Delta_{1}} C P(1)^{n}=\left(\begin{array}{l}
n \\
k
\end{array}\right) 2^{k} C P(1)^{n-k},
$$

and so it is convenient to notice the following property of binomial coefficients. We write $v_{p}(r)$ for the highest exponent of $p$ dividing the integer $r$.

Lemma Let $n=p^{m} l,(l, p)=1$, where $p$ is a prime. If $k \leqq p^{m}$ then $v_{p}\left(\begin{array}{l}n \\ k\end{array}\right)=m-v_{p}(k)$.

Proof. Simply notice that

$$
\left(\begin{array}{l}
n \\
k
\end{array}\right)=\frac{p^{m} l}{k} \prod_{i=1}^{k-1} \frac{p^{m} l-i}{i}
$$

and that $v_{p}\left(p^{m} l-i\right)=v_{p}(i)$ in this range.

Proof OF (a). We show that in $\left(N_{1}\right)_{2 n} / M U_{2 n}$ the elements of order $p$ must be multiples of $C P(p-1)^{k} / p$, where $k=n /(p-1)$ is assumed to be integral. For let $a \in\left(N_{1}\right)_{2 n}, p a \in M U_{2 n}, a \notin M U_{2 n}$. Write $a=\lambda C P(p-1)^{k}+$ $\sum \lambda_{I} H_{I}$ with $\lambda$ and $\lambda_{I}$ rational (the $H_{I}$ are monomials in the generators of $M U_{*}$ ). Since $p a \in M U_{2 n}, p \lambda$ and $p \lambda_{I}$ are integral. An argument with types (compare $[3, \S 14])$ now shows that $p \lambda_{I} \equiv 0 \bmod p$, hence all $\lambda_{I} \in Z$. Since

$$
a=p \lambda\left(C P(p-1)^{k} / p\right)+\sum \lambda_{I} H_{I},
$$

$a$ has the desired form.

Proof OF (b). A computation using the lemma shows easily that, for $\omega>0, s_{\omega}\left(C P(p-1)^{k}\right) \equiv 0 \bmod p^{m+1}$, so $C P(p-1)^{k} / p^{m+1}$ belongs to $N_{1}$. 
For $p>2$ one computes that $C P(p-1)^{k} / p^{m+1}$ has type $(p-1)$. This also holds for $p=2$ and $m=0$ (i.e., $n$ odd). For $p=2$ and $m>0$ one finds that $C P(1)^{n} / 2^{m+1}$ has type $(1,1)$; explicitly, $s_{1}\left(C P(1)^{n} / 2^{m+1}\right) \equiv C P(1)^{n-1} \bmod 2$, $s_{1,1}\left(C P(1)^{n} / 2^{m+1}\right) \equiv C P(1)^{n-2} \bmod 2$, and $s_{\omega}\left(C P(1)^{n} / 2^{m+1}\right) \doteq 0 \bmod 2$ if $\omega>(1,1)$. These observations make up Proposition 4 of [6], hence we do not offer more details.

PrOOF OF (c). Let $p=2, n$ even and $n>2$. Write $n=2^{m} l$ with $l$ odd, hence $m>0$. Using the $M U$-characteristic numbers of $H_{2,2}$ listed in Proposition 3 of [6], we learn that $s_{1} H_{3} \equiv C P(1) \bmod 2, s_{1,1} H_{3} \equiv$ $C P(1)^{2} \bmod 2$ and $s_{\omega} H_{3} \equiv 0 \bmod 2$ if $\omega>(1,1)$. It follows that

$$
s_{\omega}\left[C P(1)^{n} / 2^{m+1}+H_{3} C P(1)^{n-3}\right] \equiv 0 \bmod 2
$$

for all $\omega>0$, hence $P=C P(1)^{n} / 2^{m+2}+H_{3} C P(1)^{n-3} / 2$ belongs to $N_{1}$.

The next task is to determine the type of $P$. We establish a little more:

(d) $s_{3}(P) \not \equiv 0 \bmod 2$; if $m=1$ then $P$ has type (3), and if $m>1$ then $P$ has type $(1,1,1,1)$.

To show that (d) implies (c), we must show that it is impossible to have $s_{\omega}(P) \equiv s_{\omega}(a) \bmod 2$ for all $\omega>0$ with $a \in M U_{2 n}$. By an argument with types, this can happen only if $P$ has type $(1,1,1,1)$, i.e., $m>1$, and then $a$ must be a linear combination of $C P(1)^{n-6}\left(H_{3}\right)^{2}, C P(1)^{n-3} H_{3}$, $C P(1)^{n-2} C P(2)$, and $C P(1)^{n}$. But then we would have $s_{3}(a) \equiv 0 \bmod 2$, which violates $(d)$.

Proof OF (d) First of all one computes directly that $s_{3}(P)=C P(1)^{n-3}$, hence $s_{3}(P) \not \equiv 0$ mod 2 . Thus it remains to consider $s_{\omega}(P)$ with $\omega>(3)$.

If $\omega \neq(1, \cdots, 1)$, then

$$
s_{\omega}(P)=\frac{1}{2} s_{\omega}\left(H_{3} C P(1)^{n-3}\right) .
$$

Since $s_{\omega}\left(H_{3}\right)=0$ for dimensional reasons, $s_{\omega^{\prime}}\left(H_{3}\right) \equiv 0 \bmod 2$ if $\omega^{\prime} \neq$ $(1, \cdots, 1)$ and $s_{\omega^{\prime \prime}}\left(C P(1)^{n-3}\right)=0$ if $\omega^{\prime \prime} \neq(1, \cdots, 1)$ it follows easily that $s_{\omega}(P) \equiv 0 \bmod 2$.

So we need only consider $s^{k \Delta_{1}}(P)$ for $k \geqq 4$. One first shows that

$$
s^{k \Delta_{1}}\left\{C P(1)^{n}+2^{m+1} H_{3} C P(1)^{n-3}\right\} \equiv\left(\begin{array}{l}
n \\
k
\end{array}\right) 2^{k} C P(1)^{n-k} \bmod 2^{m+3},
$$

hence one wants to know when $v_{2}\left(\begin{array}{c}2 m l \\ k\end{array}\right)+k \geqq m+3$. E.g., if $m=1$ we want to know when $v_{2}\left(\begin{array}{l}2 l \\ k\end{array}\right)+k \geqq 4$; since $k \geqq 4$ this always holds, hence $s^{k \Delta_{1}}(P) \equiv 0 \bmod 2$ for $k \geqq 4$ when $m=1$. This proves (d) for $m=1$.

Finally let $m>1$. Note that we may assume $1 \leqq k \leqq m+2 \leqq 2^{m}$. Then the lemma implies that

$$
v_{2}\left(\begin{array}{c}
2 m l \\
k
\end{array}\right)=m-v_{2}(k) .
$$


Hence $v_{2}\left(\begin{array}{c}2 m l \\ k\end{array}\right)+k \geqq m+3$ iff $k \geqq v_{2}(k)+3$, and this fails for $k=4$, hence $s_{1,1,1,1}(M) \not \equiv 0 \bmod 2$; but it holds for $k>4$, which proves (d) for $m>1$. Q.E.D.

\section{REFERENCES}

1. V. M. Buhštaber, The Chern-Dold character in cobordisms. I, Mat. Sb. 83 (125) (1970), 575-595=Math. USSR Sb. 12 (1970), 573-594. MR 42 \#8507.

2. - Spectral sequences connected with the Chern-Dold character, Uspehi Mat. Nauk 26 (1971), 214-215.

3. P. E. Conner and E. E. Floyd, The relation of cobordism to $K$-theories, Lecture Notes in Math., no. 28, Springer-Verlag, New York and Berlin, 1966. MR 35 \#7344.

4. P. S. Landweber, Cobordism operations and Hopf algebras, Trans. Amer. Math. Soc. 129 (1967), 94-110. MR 36 \#2145.

5. S. P. Novikov, The methods of algebraic topology from the viewpoint of cobordism theories, Izv. Akad. Nauk SSSR Ser. Mat. 31 (1967), 855-951= Math. USSR Izv. 1 (1967), 827-922. MR 36 \#4561.

6. N. V. Panov, Characteristic numbers in U-theory, Izv. Akad. Nauk SSSR Ser. Mat. 35 (1971), 1356-1376= Math. Izv. 5 (1971), 1365-1385.

7. R. E. Stong, Notes on cobordism theory, Mathematical Notes, Princeton Univ. Press, Princeton, N.J.; Univ. of Tokyo Press, Tokyo, 1968. MR 40 \#2108.

Department of Mathematics, Rutgers University, New Brunswick, New JERSEY 08903 